\title{
Amplification of Frequency-Modulated Similariton Pulses in Length-Inhomogeneous Active Fibers
}

\author{
I. O. Zolotovskii, ${ }^{1}$ D. I. Sementsov, ${ }^{1}$ A. A. Sysolyatin, ${ }^{2}$ A. S. Belanov, ${ }^{3}$ and M. S. Yavtushenko ${ }^{1}$ \\ ${ }^{1}$ Ul'yanovsk State University, 42 L.Tolstogo Street, Ul'anovsk 432700, Russia \\ ${ }^{2}$ Fiber Optics Research Center, 38 Vavilov Street, Moscow 119333, Russia \\ ${ }^{3}$ Moscow State University of Instrument Engineering and Computer Sciences, 20 Stromynka Street, Moscow 107996, Russia
}

Correspondence should be addressed to D. I. Sementsov, sementsovdi@ulsu.ru

Received 4 May 2011; Accepted 21 June 2011

Academic Editor: Christophe Finot

Copyright (C) 2012 I. O. Zolotovskii et al. This is an open access article distributed under the Creative Commons Attribution License, which permits unrestricted use, distribution, and reproduction in any medium, provided the original work is properly cited.

The possibility of an effective gain of the self-similar frequency-modulated (FM) wave packets is studied in the lengthinhomogeneous active fibers. The dynamics of parabolic pulses with the constant chirp has been considered. The optimal profile for the change of the group-velocity dispersion corresponding to the optimal similariton pulse amplification has been obtained. It is shown that the use of FM pulses in the active (gain) and length-inhomogeneous optical fibers with the normal group-velocity dispersion can provide subpicosecond optical pulse amplification up to the energies higher than $1 \mathrm{~nJ}$.

\section{Intoduction}

Generation of stable self-similar FM optical pulses, referred to as similaritons, in an active (gain) medium with the normal group-velocity dispersion (GVD) opens up extensive advantages over the classical ways of an optical pulse amplification [1-16]. In the case of the optical pulse amplification in an anomalously dispersive medium, it is difficult to avoid various instabilities as well as Raman laser effect that could considerably distort the wave packet profile. However, in the normal GVD medium, there also arise some definite problems. Some known methods of the pulse amplification in homogeneous optical fibers are sensitive to the stochastic diameter variation of the mentioned fibers [17-19]. Therefore, the use of "long" amplifiers (the length $>100 \mathrm{~m}$ ) with a low gain increment $\gamma>1 \mathrm{M}^{-1}$ proves inefficient.

To overcome this problem, it is reasonable to use the compact inhomogeneous amplifiers with the normal GVD increasing over the length and gain increment of the fiber material $\gamma \ll 1 \mathrm{M}^{-1}$. In this case, the following parameters can be efficiently controlled over the optical fiber length (due to a relatively short amplifier length): the fiber core diameter, dispersion, and nonlinearity. The present work analyses the dynamics of the parabolic FM wave packet in the length-inhomogeneous amplifier and describes the conditions for their stable (self-similar) propagation in a form of the similariton wave packet.

\section{Basic Equations}

We will consider the optical pulse dynamics in the inhomogeneous amplifying medium. In this case, the field of the wave packet which evolves in an optical fiber can be expressed as

$$
\begin{aligned}
\vec{E}(t, r, z)= & \frac{1}{2} \vec{e} U(r, z) \\
& \times\left\{A(t, z) \exp \left[i\left(\omega_{0} t-\int_{0}^{z} \beta^{\prime}(\xi) d \xi\right)\right]+\text { C.C. }\right\},
\end{aligned}
$$

where $\vec{e}$ is the unit vector of the light field polarization; $U(r, z)$ function describes the radial field propagation in an optical fiber; $\omega_{0}$ represents the carrier frequency of a wave packet; $\beta^{\prime}(z)$ is the real component of the propagation constant. The nonlinear Schrödinger equation with coefficients 
inhomogeneous over the optical fiber length is valid for the time pulse envelope $A(t, z)$

$$
\frac{\partial A}{\partial z}-i \frac{D(z)}{2} \frac{\partial^{2} A}{\partial \tau^{2}}+i R(z)|A|^{2} A=g(z) A,
$$

where the following parameters are introduced: $\tau=t-$ $\int_{0}^{z} d \xi / u(\xi)$ is the time of the traveling coordinate system; $u(z)=(\partial \beta(z) / \partial \omega)_{0}^{-1}$ and $D(z)=\left(\partial^{2} \beta(z) / \partial \omega^{2}\right)_{0}$ are the group velocity and the fiber GVD; $R(z)$ is Kerr nonlinearity coefficient. The dependence of the fiber parameters on the longitudinal coordinate $z$ is associated, first of all, with their dependence on the effective mode area $S_{m}(z)$. Thus, the parameter of the mode amplification is related not only to the material gain $\gamma(z)$ but also to the mode area as well in the following correlation:

$$
g(z)=\gamma(z)-\frac{1}{2 S_{m}(z)} \frac{d S_{m}(z)}{d z},
$$

where,

$$
S_{m}(z)=2 \pi \int_{0}^{\infty}\left|U_{m}(r, z)\right|^{2} r d r
$$

is the mode area. Let us assume the dispersion and nonlinearity parameters to be the functions of $z$ coordinate which are defined as $D(z)=D_{0} d(z)$ and $R(z)=R_{0} r(z)$, where $D_{0}, R_{0}$ are the values of these parameters at the fiber input. Introduction of a new variable $\xi(z)=\int_{0}^{z} d\left(z^{\prime}\right) d z^{\prime}$ and the pulse envelope $C(\tau, z)=\sqrt{r(z) / d(z)} A(\tau, z)$ to (2) yields the equation with the constant dispersion and nonlinearity but length-inhomogeneous gain

$$
\frac{\partial C}{\partial \xi}-i \frac{D_{0}}{2} \frac{\partial^{2} C}{\partial \tau^{2}}+i R_{0}|C|^{2} C=g_{\mathrm{ef}}(\xi) C .
$$

The effective and inhomogeneous (with the respect to a new coordinate $\xi$ ) gain increment is defined as

$$
g_{\mathrm{ef}}(\xi)=\frac{\gamma(\xi)}{d(\xi)}-\frac{1}{2} \frac{\partial}{\partial \xi} \ln \frac{S_{m}(\xi)}{S_{m}(0)} \frac{d(\xi)}{r(\xi)} .
$$

Having performed all substitutions, the problem to describe the nonlinear pulse propagation in the optical fiber with such parameters inhomogeneous over the length as GVD, nonlinearity, gain and effective mode area is reduced to the description of the optical pulse propagation in the fiber with the homogeneous dispersion $D_{0}$ and nonlinearity $R_{0}$ and inhomogeneous effective gain $g_{\mathrm{ef}}$, which strongly depends on the mode profile $S_{m}(z)$.

\section{Conditions of Similariton Existence in the Amplifying Fiber}

As known, the singularity of a similariton pulse propagation consists of the fact that the pulse envelope, irrespective of its original profile, asymptotically approaches to a parabolic shape scaled along the pulse coordinate [4-9]. In this case, the pulse can be expressed as:

$$
A(\tau, z)=A(z) G_{\mathrm{par}}(z, \tau) \exp \left[i\left(\varphi(z)+\alpha(z) \tau^{2}\right)\right],
$$

where $\phi(z)$ is the pulse phase; $\alpha(z)$ is the FM rate (chirp) which is constant for a similariton. Here, function $G_{\mathrm{par}}(\tau, z)$ assigns the profile of the parabolic pulse envelope and is determined by

$$
G_{\mathrm{par}}= \begin{cases}\sqrt{\frac{1-\tau^{2}}{\tau_{s}^{2}(z)}}, & \tau \leq \tau_{s}(z), \\ 0, & \tau>\tau_{s}(z),\end{cases}
$$

where $\tau_{s}(z)$ is the pulse duration.

It is known that the condition under which (5) has an exact solution in a form of FM parabolic pulse can be expressed as [6-9]

$$
g_{\mathrm{ef}}-3 \alpha_{0} D_{0}=0 .
$$

In the case of an active optical fiber, the condition for producing a stable similariton, that is, condition (7), can be given as

$$
\gamma_{0}-3 \alpha_{0} D_{0}=0
$$

It is noteworthy that the available sources of picosecond pulses used to generate similariton FM pulses, as a rule, can produce (after passing through the complementary dispersive components) chirp of some $10^{23}-10^{24} \mathrm{c}^{-2}$. Moreover, the GVD values of the amplifying fibers being used do not exceed $3 \cdot 10^{-26} \mathrm{c}^{2} / \mathrm{M}$ (in practice, for instance, the GVD of $\mathrm{Er}^{3+}$ doped fibers, in rare cases, exceed this value $[6-8,19,20])$. As a consequence, the gain increment of the homogeneous fiber which is responsible for the FM similariton production must be appreciably lower than $0.1 \mathrm{~m}^{-1}$ (a more precise analyses yields $\gamma$ value not more than $\left.0.01 \mathrm{~m}^{-1}\right)$. In this case, to increase the pulse energy more than by an order of magnitude is required that the fiber length exceed 10 meters (and even 100 meters). As it follows from the experiments on Er-, Yb-, and Bi-doped fibers [7, 8], as well as from the numerical simulation $[18,19]$, self-similar FM pulses are very sensitive to the fluctuation of the gain increment and variation of the fiber amplifier diameter. By virtue of this fact, it proves difficult to use long (longer than 100 meters) active fibers to reach considerable (more than by an order of magnitude) pulse energy increase. The way out could be a short fiber $(<10$ meters) with a high gain $(\gamma>$ $1 \mathrm{M}^{-1}$ ) and GVD sharply increasing over its length. Further, the possibility of an efficient "rapid" parabolic FM pulse amplification will be studied in the length-inhomogeneous fiber with $\mathrm{W}$-profile of the refractive index distribution over the fiber cross-section. Taking into account expression (6), condition (9) acquires the following form for the effective gain increment:

$$
6 \alpha_{0} D(z)=2 \gamma(z)-\frac{1}{S_{m}} \frac{\partial S_{m}}{\partial z}+\frac{1}{R} \frac{\partial R}{\partial z}-\frac{1}{D} \frac{\partial D}{\partial z} .
$$

Here, the nonlinearity-dependent term can be rewritten as

$$
\frac{1}{R} \frac{\partial R}{\partial z}=\frac{1}{n_{2}} \frac{\partial n_{2}}{\partial z}-\frac{1}{S_{\mathrm{ef}}} \frac{\partial S_{\mathrm{ef}}}{\partial z},
$$


where $n_{2}$ is the cubic nonlinearity parameter, and effective mode area is introduced as

$$
S_{\mathrm{ef}}(z)=\frac{2 \pi\left(\int_{0}^{\infty}\left|U_{m}(r, z)\right|^{2} r d r\right)^{2}}{\int_{0}^{\infty}\left|U_{m}(r, z)\right|^{4} r d r} .
$$

Using the derived relations, we will arrive to the following equation describing the GVD profile over the fiber length:

$$
\frac{\partial D}{\partial z}-f(z) D(z)+6 \alpha_{0} D^{2}(z)=0,
$$

where the function $f(z)=2 \gamma(z)-\left(1 / S_{m}\right)\left(\partial S_{m} / \partial z\right)+$ $(1 / R)(\partial R / \partial z)$ is introduced.

Solution of (14) allows to obtain a required profile of the GVD variation which provides a self-similar propagating regime of the parabolic FM pulses. This equation represents a well-known Bernoulli equation, and its solution can be written in the following way:

$$
\begin{aligned}
D(z)= & D_{0} F(z) \\
& \times \frac{\exp \left[2 \int_{0}^{z} \gamma\left(z^{\prime}\right) d z^{\prime}\right]}{1+6 \alpha_{0} D_{0} \int_{0}^{z} F\left(z^{\prime}\right) \exp \left[2 \int_{0}^{z^{\prime}} \gamma\left(z^{\prime \prime}\right) d z^{\prime \prime}\right] d z^{\prime}},
\end{aligned}
$$

where $D_{0}$ is the input GVD, and the following notion is introduced:

$$
F(z)=\frac{S_{\mathrm{ef}}(0)}{S_{\mathrm{ef}}(z)} \frac{S_{m 0}}{S_{m}(z)} \frac{n_{2}(z)}{n_{20}} .
$$

If the material gain is kept constant over the whole fiber length and relation $F(z) \approx 1$ is fulfilled (that is, valid, with a high precision, for most practically important cases, and, first of all, for W-profile fibers [18-20]), the required GVD profile will have the following view:

$$
D(z)=\frac{\gamma D_{0} e^{2 \gamma z}}{\gamma+3 \alpha_{0} D_{0}\left(e^{2 \gamma z}-1\right)} .
$$

Taking into account (17), the efficient amplification of frequency-modulated pulses could be carried out when $\gamma / 3 \alpha_{0}>D(0)$ and the normal dispersion is increasing along the active fiber length. If $\gamma / 3 \alpha_{0}<D(0)$, then the dispersion should decrease along the length, and if $\gamma / 3 \alpha_{0} \approx D(0)$, the dispersion should be constant along amplifier length. It could be noted that the first case is fast scenario (the amplifier length is less than $10 \mathrm{~m}$ ) and the second is slow.

Figures 1(a) and 1(b) show the relative dispersion function along the amplifier length to reach optimal similariton amplification. The grow of similariton pulse energy is possible if the GVD is increasing essentially. The relation between the final and initial similariton energy is close to the $D(z) / D(0)$.

For a passive fiber with the negligibly low optical loss (i.e., fiber with $\gamma \rightarrow 0$ ), the GVD satisfying the condition for the similariton existence in $\mathrm{W}$-profile fiber will have a hyperbolic shape (что полностью соответствует результатам полученным в работах $[11,13])$

$$
D(z)=\frac{D_{0}}{1+6 \alpha_{0} D_{0} z} .
$$

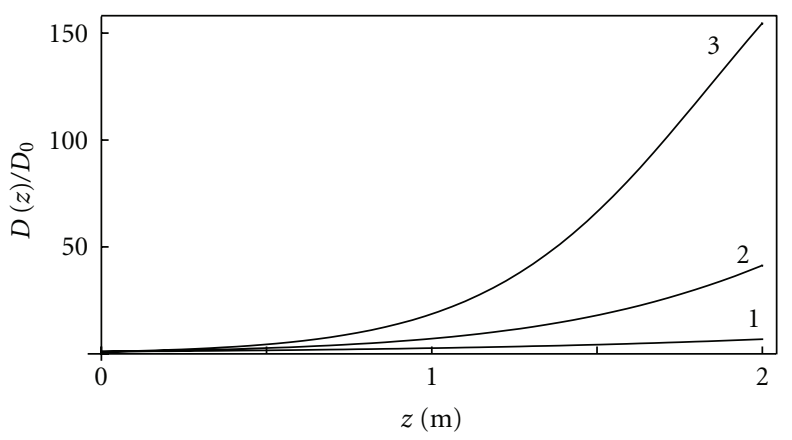

(a)

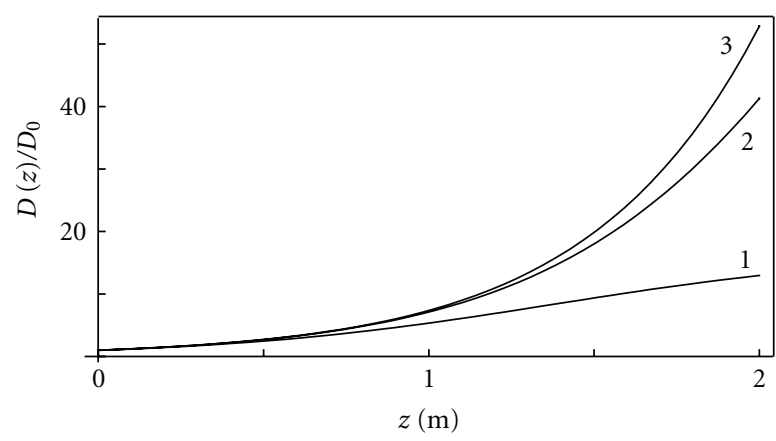

(b)

FIGURE 1: The GVD profiles providing self-similar propagation of FM parabolic pulses in the optical fiber with the following fixed parameters: $\tau_{0}=10^{-12} \mathrm{~s}, D_{0}=2 \cdot 10^{-27} \mathrm{~s}^{2} / \mathrm{m}$ and fitting parameters: $\alpha_{0}=10^{24} \mathrm{~s}^{-2}, \gamma=(0.5 ; 1.0 ; 1.5) \mathrm{m}^{-1}$ (a) and $\gamma=1 \mathrm{~m}^{-1}, \alpha_{0}=$ $\left(10^{23}, 10^{24}, 10^{25}\right) \mathrm{s}^{-2}$ (b). One can see that exponential character of GVD at $\gamma \approx 1 \mathrm{~m}^{-1}$ is kept for the fibers shorter than $2 \mathrm{~m}$.

Besides, in the general case, the following relation can be written for the similariton pulse duration [9]:

$$
\tau_{s}(z)=\tau_{0} \exp \left(2 \alpha_{0} \int_{0}^{z} D\left(z^{\prime}\right) d z^{\prime}\right),
$$

taking into account relation (15), we can express it as

$$
\tau_{s}(z)=\tau_{0}\left[1+6 \alpha_{0} D_{0} \int_{0}^{z} F\left(z^{\prime}\right) \exp \left(2 \int_{0}^{z^{\prime}} \gamma\left(z^{\prime \prime}\right) d z^{\prime \prime}\right) d z^{\prime}\right]^{1 / 3} .
$$

If $F(z)=1$ and $\gamma=0$, we obtain the following known relation for the pulse duration $[3,4]$ :

$$
\tau_{s}(z)=\tau_{0}\left[1+6 \alpha_{0} D_{0} z\right]^{1 / 3} .
$$

In the general case, the formation energy for the parabolic similariton can be defined as follows [9]:

$$
W_{s}=\frac{8 c D(0) \alpha_{0}^{2} \tau_{0}^{3}}{3 \omega_{0} n_{2}(0)} S_{\mathrm{ef}}(0) .
$$

Equations (22) and (11) describe the condition under which the stable self-similar pulses similaritons are generated. The energy change of the wave packet during its propagation through the fiber is defined

$$
W_{s}(z)=W(0) \exp \left(2 \int_{0}^{z} \gamma\left(z^{\prime}\right) d z^{\prime}\right)
$$




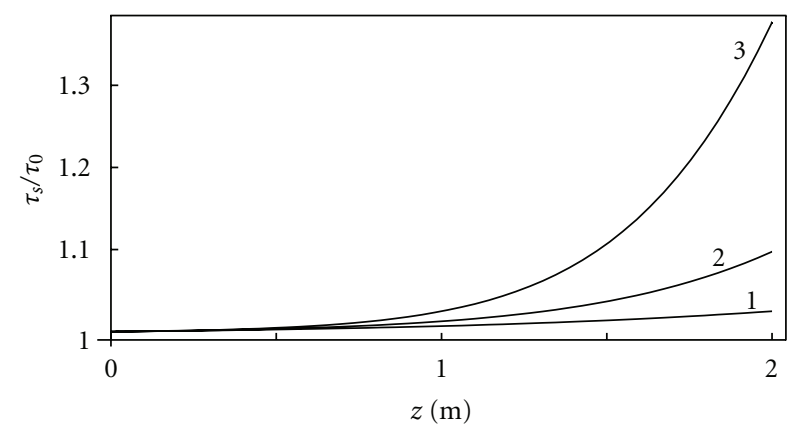

(a)

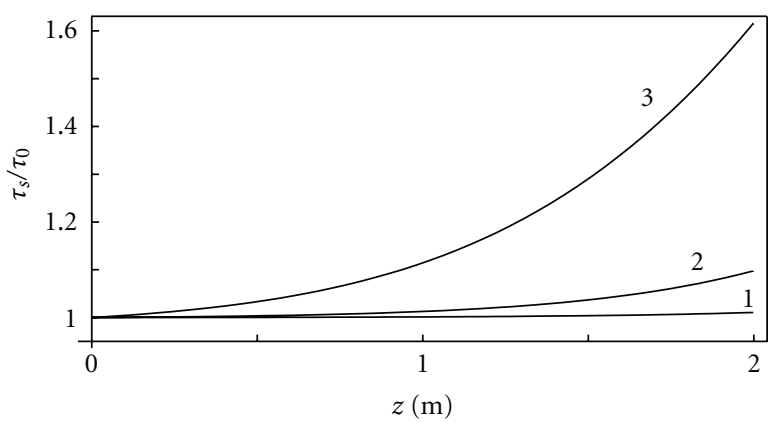

(b)

Figure 2: The normalized duration of similariton pulse versus output gain with the following fixed parameters: $\tau_{0}=10^{-12} \mathrm{~s}$, $D_{0}=2 \cdot 10^{-27} \mathrm{~s}^{2} / \mathrm{m}$ and fitting parameters: $\alpha_{0}=10^{24} \mathrm{~s}^{-2}, \gamma=$ $(0.5 ; 1.0 ; 1.5) \mathrm{m}^{-1}$ (a) and $\gamma=1 \mathrm{~m}^{-1}, \alpha_{0}=\left(10^{23}, 10^{24}, 10^{25}\right) \mathrm{s}^{-2}$ (b). One can see the pulse duration increase with the increasing output gain and fiber length.

The similariton pulse width as a function of active fiber length is shown on Figures 2(a) and 2(b) for different values of $\gamma$ and frequency modulation. For indicated fiber lengths and essential similariton energy increase, there is only a weak width change. As a result, there is a tiny spectrum modification if the chirp parameter is constant. Definitely, this condition is important for stable similariton amplification.

The stability of $g_{\mathrm{ef}}(\xi)$ should be taken into account in relation to external perturbations. It has been shown earlier [15] that the frequency-modulated parabolic pulses are stable to $g_{\mathrm{ef}}(\xi)$ variations. One could believe that the dispersion parameter would be stable too (in accordance with (6)). It is essential for efficient amplification. In addition, the influence of high-order dispersion effects is small for proposed technique of frequency-modulated pulses amplification. Indeed, if the fiber amplifier does not exceed $10 \mathrm{~m}$, the significance of the $3 \mathrm{rd}$ order dispersion would be not essential. For instance, in the case of picosecond pulse with initial chirp of $\alpha_{0}<10^{24} \mathrm{c}^{-2}$ the characteristic length $L^{(3)} \gg 10^{3} \mathrm{M}$.

\section{Compression of FM Pulses}

As follows from the performed analysis, the regime of the parabolic pulse amplification in a medium with normal dispersion is accompanied by the increase of its duration while preserving the chirp. A further increase in the peak power of the pulse due to its temporal compression should be performed in a passive dispersive medium providing a minimal nonlinear effects (it is required to avoid, as far as possible, the corresponding noises and aberration as well as the development of different kinds of instabilities [9, 10]). This procedure can be performed already outside the amplifying fiber: either in a passive anomalous dispersion fiber with a low nonlinearity (hollow fibers can be used for this purpose), or on a couple of diffraction gratings acting as an effective dispersion element. At present, this technology for producing high-energy laser pulses is the most developed [21-23]. The technology providing compression in an allfiber regime is supposed to be the method of using hollow crystal fibers with a low cubic nonlinearity [24, 25].

The duration of a bandwidth-limited pulse with the parameters $\tau_{s}(L)$ and $\alpha_{0}$ at the compressor input (after propagating through the fiber amplifier of the length $L$ ) after passing through the compressor is as follows $[9,23]$ :

$$
\tau_{\mathrm{com}}=\frac{\tau_{s}(L)}{\sqrt{1+\left(\alpha_{0} \tau_{s}^{2}(L)\right)^{2}}} .
$$

When the inequality $\alpha_{0} \tau_{s}^{2}(L) \gg 1$ is fulfilled, we have $\tau_{\text {com }} \sim$ $1 / \alpha_{0} \tau_{s}(L)$. The peak power of the compressed bandwidthlimited pulse can be determined by

$$
P_{\max } \cong P_{0} \alpha_{0} \tau_{0} \tau_{s}(L) \exp \left(2 \int_{0}^{L} \gamma(z) d z\right) .
$$

It follows from the obtained relations that the more we "stretch" the pulse with a constant nonzero chirp in time, the more compressed the pulse will be and the higher the peak power can be obtained after pulse propagation through the compressor. Thus, if the input chirp is $\alpha_{0}=10^{24} \mathrm{c}^{-2}$, the original pulse duration is $\tau_{0}=10^{-12} \mathrm{c}$, the amplifier length is $L=1 \mathrm{M}$, and the gain increment is $\gamma=2 \mathrm{M}^{-1}$, the peak power can be increased in 100 times after chirp vanish (here, according to (20), we accept that after propagating through the amplifying fiber the pulse duration becomes twice as high). The offered scheme of pulse amplification and subsequent FM pulse compression is actively used nowadays in solid-state laser systems for generating high-power pulses.

The offered scheme of pulse amplification and subsequent FM pulse compression is actively used nowadays in solid-state laser systems for generating high-power pulses.

It seems that this technique based on similariton amplification and grating compressor would allow to move forward to generation of a few cycles pulses. Taking into consideration that the similariton energy may be at the level of $10 \mathrm{~nJ}$ and above, the output peak power could be at megawatt level. Definitely, this technique may be especially fruitful in $1550 \mathrm{~nm}$ spectral region (Er-doped fiber as amplifier) $[24,25]$. Provided that increment gain $\gamma>0.5 \mathrm{~m}$, the amplifier length should be enough small (e.g., $5 \mathrm{~m}$ ) to amplify the input ps similariton more than in order. It seems the novel waveguide structure like a Bragg fiber would be useful to improve the parameters of such a all-fiber similariton amplifier. For instance, if the waveguide structure 
allows to control the GVD by 2 orders, it will result to the corresponding energy increase of FM wave packet.

At present, there is a practical way to design and produce single mode fibers with chromatic dispersion varying along the length in accordance with some prearranged low [17]. This method performs a precise control of the fiber core diameter during the drawing. This technology became possible owing to digital signal processing used for the control of the drawing process. At present, fibers with various dispersion functions can be produced, for example, dispersion decreasing or dispersion increasing. The length of fiber with varying dispersion may be in the range from several meters to several kilometers. The dispersion deviation from the prearranged value is less than $0.1 \mathrm{ps} / \mathrm{nm} / \mathrm{km}$. To design the fiber with varying dispersion, the preform with dispersion shifted waveguide structure could be used. One can obtain the relation between the chromatic dispersion and outer fiber diameter solving the scalar wave equation for the preform profile and fixed drawing coefficient. The approach to produce fibers with chromatic dispersion varying along the length in accordance with a prearranged law became possible owing to digital signal processing used for the control of the drawing process. A real-time software designed enabled measuring and remembering the process parameters during the drawing and closing a feedback loop. The software and hardware tools enabled us to control the fiber diameter during drawing. After that, the current diameter is compared with the prearranged value, and then, a control parameter (the drawing speed) was calculated in accordance with the algorithm used. Working in such a manner, the feedback system could control well of the fiber diameter. Reliable operation of the digital control system in a wide drawing speed range has allowed to fabricate single mode fibers with chromatic dispersion varying along length. The necessary length dependence of the fiber diameter was written in the memory of the control computer. During the drawing, the current fiber length is registered along with the other parameters, the current diameter was compared with the prearranged value for the given length, and the necessary change of the drawing speed is worked out by the control algorithm to produce the desirable diameter.

\section{Conclusion}

Thus, in the present paper, we have obtained the conditions for appearance of stable parabolic pulses in the optical fibers with $\mathrm{W}$-profile of radial propagation of the refractive index in the normal group velocity dispersion region. The relation for the GVD profile describing an optimally fast FM parabolic pulse amplification was offered. It was shown that the use of length-inhomogeneous FM similaritons (resistant to the wave instability) with the slightly increasing normal groupvelocity dispersion allows to produce fiber laser systems with a high peak power $P>1 \mathrm{MW}$.

\section{Acknowledgment}

This work was supported by the Federal Agency on Science and Innovations within the framework the Federal Task
Program "Academic and Teaching Staff of Innovative Russia in 2009-2013".

\section{References}

[1] M. E. Fermann, V. I. Kruglov, B. C. Thomsen, J. M. Dudley, and J. D. Harvey, "Self-similar propagation and amplification of parabolic pulses in optical fibers," Physical Review Letters, vol. 84, no. 26, pp. 6010-6013, 2000.

[2] G. Chang, H. G. Winful, A. Galvanauskas, and T. B. Norris, "Self-similar parabolic beam generation and propagation," Physical Review E, vol. 72, no. 1, Article ID 016609, pp. 1-4, 2005.

[3] J. M. Dudley, C. Finot, D. J. Richardson, and G. Millot, "Selfsimilarity in ultrafast nonlinear optics," Nature Physics, vol. 3, no. 9, pp. 597-603, 2007.

[4] T. Hirooka and M. Nakazawa, "Parabolic pulse generation by use of a dispersion-decreasing fiber with normal groupvelocity dispersion," Optics Letters, vol. 29, no. 5, pp. 498-500, 2004.

[5] A. I. Latkin, S. K. Turitsyn, and A. A. Sysoliatin, "Theory of parabolic pulse generation in tapered fiber," Optics Letters, vol. 32, no. 4, pp. 331-333, 2007.

[6] A. Y. Plotskii, A. A. Sysoliatin, A. I. Latkin et al., "Experiments on the generation of parabolic pulses in fibers with lengthvarying normal chromatic dispersion," Zhurnal Eksperimental'noi i Teoreticheskoi Fiziki, vol. 85, no. 7, pp. 397-401, 2007.

[7] A. V. Andrianov, S. V. Muraviov, A. V. Kim, and A. A. Sysoliatin, "Generation of optical soliton pulses smoothly tunable in a wide frequency range in silica fibers with variable dispersion," JETP Letters, vol. 85, no. 8, pp. 364-368, 2007.

[8] I. O. Zolotovskii, D. I. Sementsov, A. K. Senatorov, A. A. Sysolyatin, and M. S. Yavtushenko, "Dynamics of similariton pulses in length-inhomogeneous active fibres," Quantum Electronics, vol. 40, no. 3, pp. 229-233, 2010.

[9] A. S. Zeytunyan, K. A. Palandjan, G. L. Esayan, and L. K. Muradyan, "Nonlinear dispersive similariton: spectral interferometric study," Quantum Electronics, vol. 40, no. 4, pp. 327-328, 2010.

[10] J. Laegsgaard and P. J. Roberts, "Influence of air pressure on soliton formation in hollow-core photonic bandgap fibers," Journal of the Optical Society of America B, vol. 26, no. 9, pp. 1795-1800, 2009.

[11] S. Wabnitz and C. Finot, "Theory of parabolic pulse propagation in nonlinear dispersion-decreasing optical fiber amplifiers," Journal of the Optical Society of America B, vol. 25, no. 4, pp. 614-621, 2008.

[12] C. Dai, Y. Wang, and J. Zhang, "Analytical spatiotemporal localizations for the generalized (3+1)-dimensional nonlinear Schrödinger equation," Optics Letters, vol. 35, no. 9, pp. 14371439, 2010.

[13] C. Finot, B. Barviau, G. Millot, A. Guryanov, A. Sysoliatin, and S. Wabnitz, "Parabolic pulse generation with active or passive dispersion decreasing optical fibers," Optics Express, vol. 15, no. 24, pp. 15824-15835, 2007.

[14] D. Ghosh, M. Basu, and S. Sarkar, "Generation of self-similar parabolic pulses by designing normal dispersion decreasing fiber amplifier as well as its staircase substitutes," Journal of Lightwave Technology, vol. 27, no. 17, pp. 3880-3887, 2009.

[15] V. I. Kruglov, A. C. Peacock, J. M. Dudley, and J. D. Harvey, "Self-similar propagation of high-power parabolic pulses in optical fiber amplifiers," Optics Letters, vol. 25, no. 5-24, pp. 1753-1755, 2000. 
[16] C. Finot, J. M. Dudley, B. Kibler, D. J. Richardson, and G. Millot, "Optical parabolic pulse generation and applications," IEEE Journal of Quantum Electronics, vol. 45, no. 11, Article ID 5308680, pp. 1482-1489, 2009.

[17] V. A. Bogatyrjov, M. M. Bubnov, E. M. Dianov, and A. A. Sysoliatin, "Advanced fibres for soliton systems," Pure and Applied Optics, vol. 4, no. 4, article 009, pp. 345-347, 1995.

[18] U. G. Akhmetshin, V. A. Bogatyrev, A. K. Senatorov, A. A. Sysolyatin, and M. G. Shalygin, "New single-mode fibres with the flat spectral dependence of the chromatic dispersion varying over the fibre length," Quantum Electronics, vol. 33, no. 3, pp. 265-267, 2003.

[19] M. E. Likhachev, M. M. Bubnov, K. V. Zotov et al., "Erbiumdoped aluminophosphosilicate optical fibres," Quantum Electronics, vol. 40, no. 7, pp. 633-638, 2010.

[20] D. A. Grukh, V. A. Bogatyrev, A. A. Sysolyatin, V. M. Paramonov, A. S. Kurkov, and E. M. Dianov, "Broadband radiation source based on an ytterbium-doped fibre with fibre-length-distributed pumping," Quantum Electronics, vol. 34, no. 3, pp. 247-248, 2004.

[21] G. A. Mourou, T. Tajima, and S. V. Bulanov, "Optics in the relativistic regime," Reviews of Modern Physics, vol. 78, no. 2, pp. 309-371, 2006.

[22] V. V. Lozhkarev, S. G. Garanin, R. R. Gerke et al., "100TW femtosecond laser based on parametric amplification," Zhurnal Eksperimental'noi i Teoreticheskoi Fiziki, vol. 82, no. 4, pp. 196-199, 2005.

[23] S. A. Akhmanov, V. A. Vysloukh, and A. S. Chirkin, Optics of Femtosecond Laser Pulses, AIP, New York, NY, USA, 1992.

[24] D. N. Papadopoulos, Y. Zaouter, M. Hanna et al., "Generation of 63 fs $4.1 \mathrm{MW}$ peak power pulses from a parabolic fiber amplifier operated beyond the gain bandwidth limit," Optics Letters, vol. 32, no. 17, pp. 2520-2522, 2007.

[25] D. J. Richardson, J. Nilsson, and W. A. Clarkson, "High power fiber lasers: current status and future perspectives," Journal of the Optical Society of America B, vol. 27, no. 11, pp. B63-B92, 2010. 

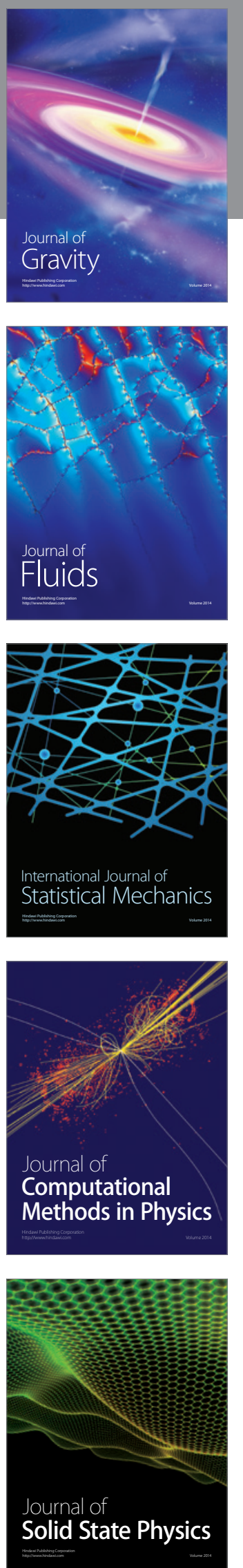

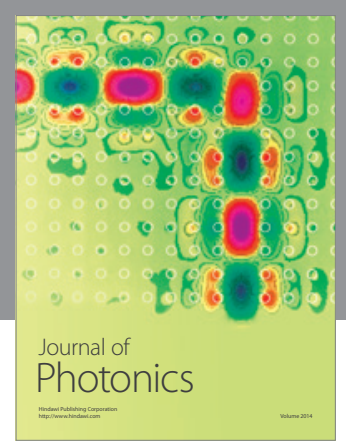

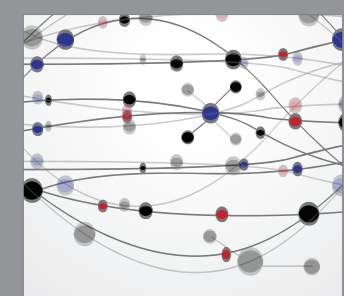

The Scientific World Journal
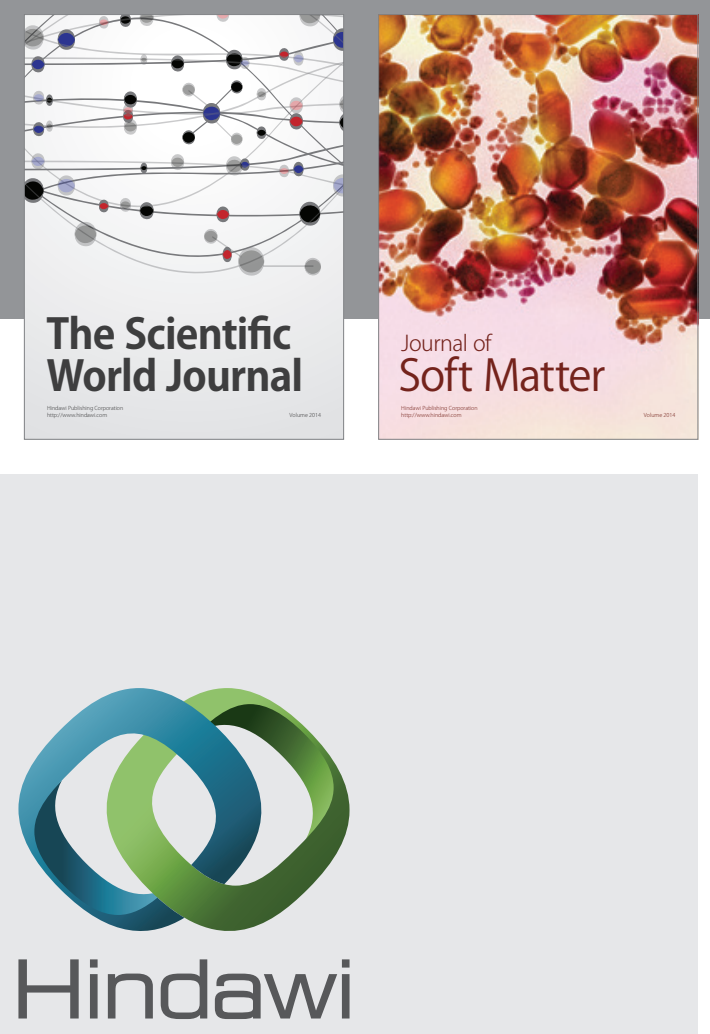

Submit your manuscripts at

http://www.hindawi.com
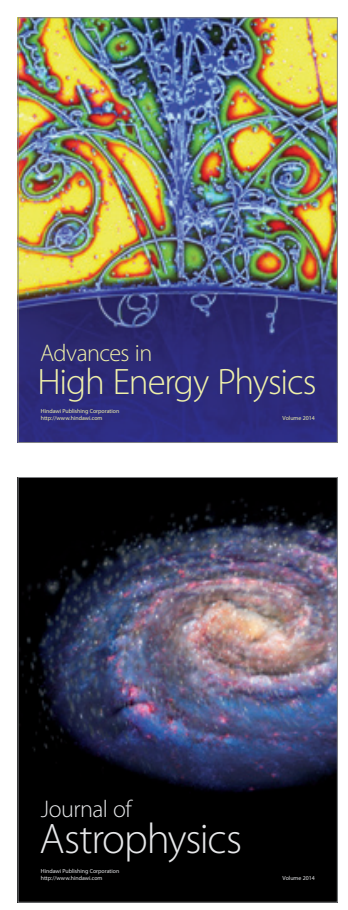
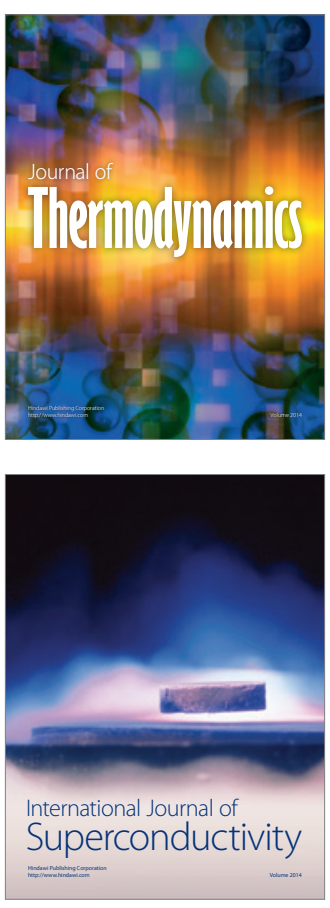
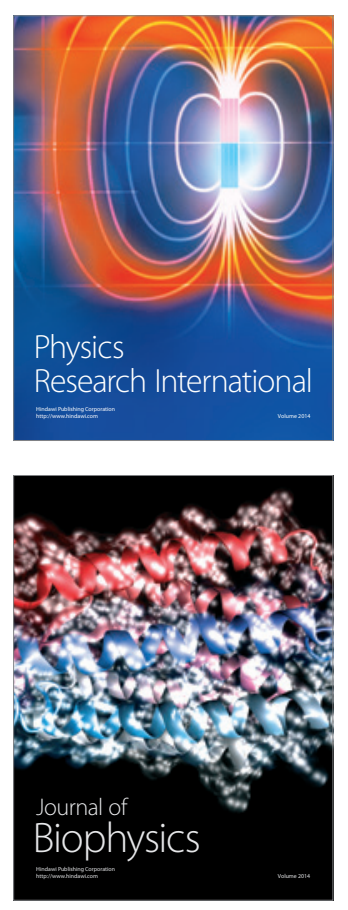
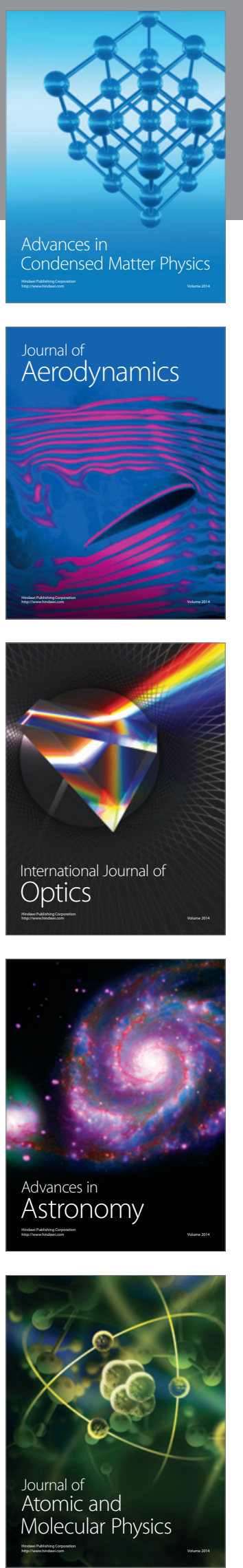